\title{
Non-Verbal Communication Means in the Contemporary Art
}

\author{
Svitlana Derkach ${ }^{1}$, Myroslava Melnyk ${ }^{1}$, Volodymyr Fisher ${ }^{1}$, Volodymyr Moiseienko ${ }^{1}$, Tetiana Sovhyra ${ }^{1}$ \\ ${ }^{1}$ Department of Variety Directing and Mass Festivals, Kyiv National University of Culture and Arts, Kyiv, Ukraine \\ Correspondence: Myroslava Melnyk, Department of Variety Directing and Mass Festivals, Kyiv National University of \\ Culture and Arts, 01133, 36 Yevhen Konovalets Str., Kyiv, Ukraine. E-mail: mmelnyk@ tanu.pro
}

Received: December 13, 2021

Accepted: January 15, 2022

Online Published: January 25, 2022

doi:10.11114/smc.v10i1.5462

URL: https://doi.org/10.11114/smc.v10i1.5462

\begin{abstract}
The relevance of this work is due to the significant spread of various concepts on the basic ways of generating diverse, modern shows of the entertainment genre with a wide range of non-verbal means of communication. Non-verbal communication means serve as mediators in art but due to the fact that in contemporary art, the communication between the audience and the author is not sufficiently arranged, there are gaps in the information message perceiving. The authors aimed to study the communication barriers related to non-verbal communication means, as well as to find the solutions for the problems that arise in this context. The following methods were used: analysis and synthesis, comparison, descriptive method, method of abstraction. Trends in cooperation developing between the authors and the subjects of receiving information were identified. The psychological and social researches' data on the different signals impact on the information receiving efficiency were analyzed. Features and characteristics of the non-verbal communication means, their relationships, similarities and differences were identified. As a result of the research, the basic concepts of creating a show in the XXI century with the involvement of non-verbal communication, their effectiveness in the development of the industry at this stage, as well as possible directions for future development were described.
\end{abstract}

Keywords: non-verbal forms of interaction, verbal and non-verbal components, contemporary art, communication barrier

\section{Introduction}

Currently the issue of various communication forms in the contemporary art is quite acute, due to the lack of studying and covering this topic in cultural, psychological and social science. It is worth noting that human communication is mostly non-verbal since humans react to numerous non-verbal cues and behaviors, including posture, facial expressions, eye contact, gestures, tone of voice. It is often used in media and shows. This issue is an integral part of choreographic art as well. Due to the lack of comprehensive scientific researches on contemporary choreography and the dance communication function in science, this issue was studied by means of Russian, Italian, American and other scientists' studies. For example, in Russian scholars research it was found that in European countries while contemporary choreography teaching, the great importance is given to self-expression and self-identity during productions' preparing (Bakirova et al., 2021).

The research by Italian scientists has proved that such factors as speed, movement, dance pace affect the information message perceiving (Orlandi et al., 2020). The results obtained in this research were confirmed by British scientists (Karageorghis et al., 2019). The research results confirm that this issue requires extensive study in terms of psychology, neuropsychology and other sciences (Christensen et al., 2021). For example, Finnish researchers in their papers pointed out the need in dancers' self-expression in contemporary choreography, which is very problematic while creating a production with a complex abstract plot. These papers consider an attempt to see different types for information transmitting non-verbally that was also studied by Finnish researchers (Anttila et al., 2019). Austrian scholars research confirms that despite the cultural diversity of dance movements, musical means and contexts, their basic communicative functions may be the same for different societies, thus indicating the cross-cultural influence of non-verbal communication means (Fink et al., 2021).

This paper also pays attention to choreographic means and specifies that choreography usually includes a combination of certain gestures with vocalization, which is common for wild animals as well since it serves for better interaction (Miles \& Fuxjager, 2018). Similarly, cognition occurs via dance, which improves perception and motional communication among dancers. Despite historical and cultural changes, expressive human movement prevails. People 
are able to appreciate and admire dance. Dance is a represented language, capable of expressing the most abstract truth and the deepest emotional experience (Somov, 2013). Owing to its unique ability to evoke kinesthetic empathy, choreography in environment-oriented productions serves as one of the efficient means of inducing the environmental empathy and influencing the environmental identity forming (Belyakova \& Mileshko, 2020). The research within this area were also conducted by American scientists studying the cognitive sides of contemporary dance movements (Carter, 2020; Pakes, 2021). The aforesaid studies are of great importance for the issue being considered for they make a practical contribution to solving the challenges related to the communication barriers in contemporary choreography.

The need to take into account non-verbal contexts and subtexts in live communication and in art, as well as the need to properly use non-verbal means to achieve specific goals, determines the relevance of the study. An important issue is the quality of presentation of the creator's ideas to the recipient, i.e. the audience. However, the study of the effects of both types of communication and the process that leads to this impact should be comprehensive, which will allow all participants of the communicative process to mutually beneficial use a combination of verbal and non-verbal means and create conditions for accurate perception of non-verbal means. The studying of the process of creating a show considering the means of nonverbal interaction is relevant and significant in the context of further development of the entertainment industry and quality content directly to each viewer. The relevance of the article issue is due to the fact that every year the trend of contemporary choreography complicating and upgrading only increases. This leads to an increase in the information gap between the audience and the author. In order to prevent the communication issue related to contemporary art including choreography it is required to investigate the increasing layer of information not only in cultural studies, sociology, but also in other sciences.

The research is novel in that it involves comprehensive studying the theoretical approaches of various authors to the aforesaid issue, as well as the empirical experience of its applying. The main elements of scientific novelty comprise preparing the suggestions and recommendations for preventing the communication barriers between the author and the audience.

\section{Method}

The research applies methods of comparative analyzing the nonverbal communication approaches while establishing common understanding between the show stage manager and the audience. The comprehensive material studying approach was applied. Considerable attention was paid to the peculiarities of the functioning of non-verbal communication and they were compared with the properties of verbal communication. The specifics of non-verbal means use in interaction with the audience are taken into account. The research logic is to consider dance as an art of conveying an information message via body language to the person. The method of abstraction was used during which non-verbal means such as facial expressions, posture, gestures, intonation, tactile contact between participants, distance, spatial orientation, appearance, gaze were identified. Features, properties and relationships in the structure of the elements of the modern show were identified on the basis of methods of synthesis and analysis. Descriptive method was used to present the results.

The first research phase allows us to understand the nature of non-verbal communication including dance-based communication form. It was revealed that the first dance movements were simple, based on natural phenomena and animal behavior. This was expressed in such elements as zoomorphism, the use of circular and oval movements. Contemporary choreography differs from primitive dances in its awareness and focus on self-expression of the dancer, which subsequently introduced many genres. At the first phase, the main research method was historical and systematic, based on the works by famous Russian and American historians and sociologists (Fink et al., 2021).

The second research phase examined the various forms of communication through which the authors can interact with the audience. Based on the second phase analysis, the following main conclusions were highlighted: firstly, in contemporary choreography the most striking form of interaction is non-verbal body language of the dancer, which can convey to the audience the most subtle and symbolic meanings. Secondly, the non-verbal communication form includes a fairly extensive range of signals that the audience subconsciously picks up and interprets, such as dancers' positioning, speed of movement, music tempo and beat, facial expressions and gestures.

The third phase identified the communication barriers faced by authors while contemporary choreography creating, as well as ways to overcome them. The main method at this phase was induction and comparison. The main studies by American, German, Italian and Russian scientists in the context of various factors influencing the attention and the information perception were studied. Based on the data the following conclusions were made: firstly, the main communication barriers were identified, such as: the collision of two personalities, the wrong form of information transferring, too much identity in the production, which are a barrier between the author and the audience. Secondly, it is required to remember that choreographic techniques, forms and means should help to create an image understandable to the audience. Thus, in order to convey the dance meaning, it is required to notice the slightest changes within the 
dance, such as improvisation, plasticity of the dancers, sound accompaniment. Thirdly, a concept of overcoming this issue is suggested, based on the theory that the audience is a full-fledged participant in the process of comprehending the performance via various symbols and meanings.

\section{Results and Discussion}

In modern society, the issues of studying the contemporary art and its influence on people are relevant, because a person is constantly within the information ecosystem, interacting with a large flow of symbols, transmitting via verbal and non-verbal communication forms the idea, interpretation of phenomena and processes. In a broad global context, a show is an event or performance of an entertaining nature that is shown to the public in one way or another. It was with the advent of television that the show became widespread and popular and profitable. In the research, choreography show as an art of composing and staging, is expressed via dance (Dyakonova, 2011). Different historical periods are specified by their own ways of communication in the form of certain movements. The information itself can be represented via kinetics, facial expressions, plastique. Thus, the historical aspect within the issue being investigated is important for understanding the communicative activity manifested in dance show, which helps to convey socio-cultural norms and values. In the paper, the issue related to the communication in contemporary art is considered within the cultural system and historical retrospective (Kharakhonova, 2018; Romanova, 2020).

Communication occupies a central place in the life of every person since most of their working or free time is spent on communication. In general, communication is the information exchange between individuals by using a common system of symbols (Sokol, 2007). While communicating the information is transferred from one person to another. Thus, the relevance of communication is growing in all spheres of life, including economics, art, entertainment industry etc. Modern science specifies three forms of communication:

1. Verbal communication, using vocal apparatus;

2. Non-verbal communication by means of language of gestures, body, movements.

3. Paraverbal communication, which combines the speech with additional intonation.

In the research, nonverbal forms of communication of the audience and the choreographer within the contemporary dance scopes were studied. An example of non-verbal communication can be the use of special effects in modern films that affect the overall impression of the viewer but require the availability of digital and other technologies and their continuous improvement.

The language of dance is specified by the human body, so the human body acts as the material and spiritual basis of dance movements (Narozhnaya, 2017). The human body moving in the dance is closely connected with its spiritual life and can directly express the inner world of the dancer. The communicative orientation of the dance movements is caused by the non-verbal rhythmoplastic thinking (Kharakhonova, 2018). Therefore, it is especially important for a choreographer to study the non-verbal contact issue because dance is his instrument to convey to the audience the artistic meaning of what is happening on stage, especially if the author fills the dance with symbolism and abstraction.

Non-verbal signs include a wide range of interaction modes that are used in contemporary choreography. For example, nonverbal communication includes:

1. Kinesics - a set of gestures, poses, body movements;

2. Tacesics - touching your partner's body;

3. Sensorics - sensory perception;

4. Proxemics - ways of using space in the communication process;

5. Chronemics - ways of using time in the communication process;

6. Oculistics - use of eye movement or eye contact in the communication process.

It can be argued that previous studies have often considered the verbal and non-verbal components of communication separately, independently. There is an opinion that these concepts should be considered as opposite, polar. However, the authors adhere to the approach, where the concepts, on the contrary, complement each other and, accordingly, should be studied holistically, because verbal and non-verbal behavior are interrelated phenomena. In this context, non-verbal communication is the transmission of information through body language, including eye contact, facial expressions, gestures, posture, position in relation to another or others, tone and timbre, voice speed, posture, body dynamics, level distances between interlocutors, tactile touches, which each individual uses consciously or subconsciously.

Following the huge layer of informational ways of communicating one's world-view to another person, we state that communicative function of dance allows us to be one of the communication means, not only intrapersonal, but also intercultural. The sacred meaning of any contemporary choreography production is to convey a meaningful message to the 
audience that perceives it. In order to perceive the information contained in the contemporary choreography production, the author is to take into account the circumstances in which he finds himself. Otherwise, he cannot avoid communication barriers - difficulties while information transferring from the person to the audience due to various circumstances.

The first communication barrier is the two minds' confrontation. This confrontation is expressed in the author's inability to convey his thoughts and meanings to the audience due to various circumstances. The most common issue is the unpreparedness of the audience to perceive complex choreographic movements. Often, the stage manager himself uses symbolism while dance creating, thus transferring this information to an unprepared audience. This is due to the fact that the author himself is not ready to compromise with the audience, considering his production of unique and complex nature. As a consequence, there is the dance interpreting issue. In order to eliminate the first communication barrier, the author should perceive the audience as an equal participant of the production, as it is on his emotions, feelings and the correct interpretation, the final result - the information perceiving - depends. Therefore, it is required to communicate with the audience via the mass media, booklets to decipher the hidden meanings and explain some abstractions.

The second communication barrier is the choice of the wrong information transferring form. It should be acknowledged that some productions cannot be expressed via contemporary choreography. For example, a new way of looking at old productions may alienate the audience due to the incorrect information presenting and delivery. It can also be the case that the stage manager works in his or her unique style however the production cannot be conceived because of the author's peculiarities. This problem also involves selecting the dancers, who are to be in line with the requirements of professional competence and mental health that would match the production.

The third communication barrier is related to the "personality". The choreographer, the audience, and the dancers are all important in the production. If the choreography is distinguished by a complex plot, improvisations on the part of dancers are possible, which in its turn may change the original conception of the author. Thus, it is possible to state that the production's author is to: at first, accept the audience as a full participant in the action; secondly, do his/her best in order to convey his idea to the audience.

As the audience consciousness is to be ready to reinterpret what is happening on stage. In contemporary art, works of art act as an object for the audience, capable not only of conveying the vision of another person's world, but also of stimulating their own consciousness to a new comprehension of objects and phenomena. In view of the aforesaid, we can conclude that communication barriers occur when there is a dissonance of transferred information from the author to the person perceiving it. This problem can be solved in the following way:

1. The author's awareness of the primary role of the audience in the production, as it is they who must process the production, draw conclusions for themselves and discover new meanings;

2. The unskilled audience's studying the symbolism and abstractions in the dance;

3. The author's interest to popularize complex productions through communication channels, explaining them to both adult and children audience;

4. To combine form and content of the production for the communication barriers' eliminating.

The communication issues in contemporary choreography have not had enough discourse in science. Although, there are a huge number of various trends of dance motives in the modern world, which are specified by abstraction, symbolism and require more comprehensive preparation for the audience. The main communication channel and form of manifestation in the dance are the gestures that can reflect the mindset and world perception of different peoples. According to the Russian art historian, owing to the dance and plastique language of each nation, watching a small dance routine expressed by several national movements, you can determine what dance it is: Russian, Georgian, Indian, Uzbek, Kazakh, even without knowing its content or just by watching it as a silent movie without music being played (Narozhnaya, 2017). This content is conveyed by means of gestures, which are communicative signs of non-verbal communication.

Another Russian author notes the special importance of gestures while dance communicating. In a verbal context, a gesture has a message function. In terms of dance, a gesture is an element of narration; the language of artistic gesture is the basis of body expression style. Within the dance communication gestures and facial expressions merge while choreographic image creating. Music complements the choreographic image, it dictates the rhythm, and the rhythm primordiality materializes the gesture (Pimenova, 2015).

If we consider ballet gestures that denote any action or emotion: love, anger, hatred, we note that most of them will be the same for the understanding among different cultures. Therefore, the dance language is universal for intercultural understanding. Communication in choreography is also achieved by means of the unified teaching method. Thanks to it, dancers can interact with different cultures when international festivals of social dance take place (Tkachuk, 2012; Mileshko, 2018). 
Being a non-verbal system, the art language has an intermediate function. However, the significance of the dance language is that dance is non-national, universal in its linguistic parameters, does not need translation, and, therefore, is able to perform a unifying function (Camurri, 2009).

The socio-cultural trends, both of body and spirit, included in dance allow it being of essence in the individual socialization process, contributing to the full comprehension of the culture world. Therefore, the non-verbal form of communication is crucial for the individual involving into the social relations system. Since the symbolism and non-verbal specificity of the dance language determine its belonging to the intense communication, in which a person reveals himself not partially, however his essence comprehensively, dance is an efficient method for overcoming people estrangement. In this sense, dance has not only an aesthetic side, but also a practical one, and is inter-phenomenon (Narozhnaya, 2017).

The function of self-identification, self-expression and individual qualities' message through body movement is very important (Orlandi et al., 2020; Bakirova et al., 2021). Dancing is widespread among people and attracts the attention of several disciplines. Ethnographic documentation suggests that dance serves a signaling function in social interaction. It can influence partner preferences and facilitate social connections. Research has provided insight into the proximate mechanisms of dance, individually or while dancing with partners or in groups (Fink et al., 2021).

There have been other similar studies on this issue, such as German researchers in cognitive psychology and affective neuropsychology trying to measure the accuracy with which human observers detect fake and genuine emotional expressions. Great part of the research has focused on facial emotions' displaying, and the results prove that observers are indeed aware of the subtle differences between genuine and fake (or pretend) facial emotion expressions. The art of dancing uses this communicative power of the human body to express emotions only via movement. Therefore, the stimuli of the dance video are explored here as a means of testing the audience accuracy while detecting the authentic emotional expression of whole-body movement.

Everyday emotion expressions by means of the body that are used as stimuli in empirical studies unfortunately contain culturally clearly recognizable signals of emotional expression (e.g., shaking the fist for anger, stooping head for sadness, etc.), whereas stimuli for expressive movements can be designed to contain completely abstract movements (i.e., movements that have no clearly distinguishable meaning). Thus, expressiveness is the result of subtle variations in the expressive person's body movements, and emotions cannot be recognized by observers through specific actions (e.g., shaking a fist).

Thus, for example, a single simple hand raise can have many different expressive meanings, including functional meanings such as reaching, touching, resistance, or greeting. Emotional expressiveness is also possible. With the same movement, sadness, anger, joy, etc. can be expressed. It always depends on how the movement is performed. Subtle variations in movement parameters matter. In view of the aforesaid, it seems feasible that we use the artistic form of dance (the type of expressive body movement) to investigate whether audience accurately distinguish truly expressive body movements from those ones that are not expressive. This issue is relevant to a wide range of scientific disciplines, including psychology of emotions and affective neuropsychology, but could also potentially be relevant to practicing specialists such as audience researchers, dance teachers, and choreographers (Christensen et al., 2021).

Thus, the information perceiving while intercultural dance communicating is possible at the nonverbal communication level via a system of signs (iconic gestures), at the level of emotions via the artistic images perceiving, and at the level of kinesthetic empathy. This universality of perception can be applied in solving global planetary problems, such as environmental ones. Due to its unique ability to evoke kinesthetic empathy, choreography with regard to the environmentally oriented productions serves as one of the efficient means for evoking the ecology empathy and influence the ecology identity occurring (Belyakova \& Mileshko, 2020).

At the moment contemporary choreography is a flexible, changeable form that responds to the modern pace of living. It is a form that is flexible, constantly changing, subject to a large number of influencing factors.

In Russian culture, choreography focuses on the technical side of dancing, leaving behind the important connection with the audience. With regard to the classical dancing, as mentioned above, it is required to understand and follow each movement, which will give the person some information. For example, in Czech Art Universities, students are taught dancing by means of using an augmented reality system, owing to which, they memorize the movements better and perform the task more clearly. It should be kept in mind that communicative actions between the audience and the author are only possible via dialogue. Finnish researchers also tried to see different forms of transmitting information via dance. They found that the dance context is related to the space and ideological content of dance, within which the dancers can express themselves (Anttila et al., 2019).

Based on the research by Russian, American, Italian and other scientists the following conclusions can be made: 
1. For proper art meaning transferring, both the whole plot and the individual movements, the author, as the creator of the information being conveyed, should pay his attention to the communication that occurs between the performers within the process. The slightest changes, such as improvisation, should not be allowed since they can change the entire content.

2. The author can make mistakes. For example, excess abstraction and symbolism using, in order to surprise the audience, leaving behind the primary objectives.

3. Any dance requires musical accompaniment, and as noted in studies by Italian scientists, the rhythmic movements and properly selected music will contribute to the harmonious information transmitting. Therefore, it is impossible to consider a performance without considering the musical form and figurative content. Thus, when staging a choreographic performance, the author will also have to reveal the musical image created by the composer, using his own choreographic means. Therefore, the music selecting is an important communication means (Volkova, 2016).

4. It is to be kept in mind that choreographic techniques, forms and means should help to create an understandable image for the audience. For example, many critics have noted that the Spanish folk dance performed in Tchaikovsky's ballet Swan Lake to stylized Spanish music at a fast tempo should not be for pointe (Fokin, 1962).

5. The choreographer, in order to achieve the set objectives, should also take into account the following point. For example, the selecting the dancers by age, sex, character, physical data to implement the most complex dance movements. For example, in "Moulin Rouge" (2001) movie directed by Baz Luhrmann, the dance of girls from the variety show was performed by fourteen- and sixteen-year-old schoolgirls, what resulted in criticisms. The director has not foreseen that the movements of some performers were awkward, they lack understanding of the poses' meaning they perform and, consequently, the performance was formal (Buzskaya, 2016). Thus, the director in his work did not provide for psychological and physiological factors affecting the performance quality, which in its turn formed a barrier in communication with the audience.

6. The stage manager should also keep in mind that the costume has a great semantic meaning. And on stage there can be no casual details. Unfortunately, the choreographer may encounter trivial current problems, namely the lack of material resources, which does not allow the choreographer to implement completely his idea (Horrigan, 2020).

\section{Conclusions}

The topic of verbal and non-verbal communication is presented, both types of communication were presented as a holistic object for research without contrasting verbal and non-verbal components. Analyzing the authors and audience functioning within the contemporary art scopes, it should be specified that in the art modernization era, the dance movements complicating as well as filling with abstractions and symbolism are inevitable, which in their turn results in misinterpretation of certain choreographer intentions. As it was stated in this research, body language carries a whole set of unique signals that our subconscious picks up and can identify under certain situations in different ways.

As studies in cultural studies, history, neuropsychology prove, absolutely all nonverbal signals, can be perceived by the person who is receiving the information in different meanings. Historically, the first dance movements in different world cultures have been identified as ways of communication between people. Nonverbal communication forms, as well as their types in terms of the information transferring efficiency were specified. The communication barriers in contemporary art that can be faced by the author and the audience were identified. The researches by Russian, American, Finnish and other scientists with regard to neuropsychology, cultural studies and sociology areas were analyzed. Based upon the aforesaid, the following results were obtained: the influence of dance tempo and rhythmics on perceiving the production meaning, studying the modern approaches to choreography teaching, the dance-related communication issues origins. The use of non-verbal means in the process of creating a modern show and their growing relevance in the progressive world was highlighted.

Based on the theoretical and practical material, the following changes should be introduced for solving the contemporary dance-related communication issues:

1. In order to avoid production misinterpreting, the author is to cooperate with the audience by explaining complex abstract concepts and symbols.

2. The correct production content narrating form and the most appropriate dancers who will be able to perform the intended, in terms of skills and psychophysiological understanding should be selected.

3. the unique style of the author should be combined with musical accompaniment, understanding the historical context of the production, the level of dancers and the audience competence to whom the choreography will be conveyed.

The materials of this article can be useful for authors, speechwriters, teachers and students who are involved in such areas as journalism, cinema, cultural studies and others. 


\section{References}

Anttila, E., Martin, R., \& Svendler, N. C. (2019). Performing difference in through dance: The significance of dialogical, or third spaces in creating conditions for learning and living together. Thinking Skills and Creativity, 31, $209-216$. https://doi.org/10.1016/j.tsc.2018.12.006

Bakirova, S. A., Izim, T. O., Nikolayeva, L. A., \& Saitova, G. Y. (2021). Choreographic art features: Creative concepts and innovations in teaching. Thinking Skills and Creativity, 41, article number 100901. https://doi.org/10.1016/j.tsc.2021.100901

Belyakova, I. G., \& Mileshko, A. L. (2020). Art and sustainability. Formation of environmental identity by means of choreography. Observatory of Culture, 17(2), 187-200. https://doi.org/10.25281/2072-3156-2020-17-2-187-200

Buzskaya, O. M. (2016). Dance as a form of communication between the cultures of Russia and Ireland. Vestnik of the Plekhanov Russian University of Economics. Introduction. The Path to Science, 1, 135-141.

Camurri, A. (2009). Non-verbal full body emotional and social interaction: A case study on multimedia systems for active music listening. Lecture Notes of the Institute for Computer Sciences, Social-Informatics and Telecommunications Engineering, 9, 9-18. https://doi.org/10.1007/978-3-642-02315-6_2

Carter, C. 2020. Dance and choreography as a method of inquiry. Forum: Qualitative Social Research, 21(3). https://doi.org/10.17169/fqs-21.3.3448

Christensen, J. F., Azevedo, R. T., \& Tsakiris, M. (2021). Emotion matters: Different psychophysiological responses to expressive and non-expressive full-body movements. Acta Psychologica, 212, article number 103215. https://doi.org/10.1016/j.actpsy.2020.103215

Dyakonova, L. T. (2011). Dance as a cultural phenomenon. Society. Environment. Development, 3, 155-158.

Fink, B., Bläsing, B., Ravignani, A., \& Shackelford, T. K. (2021). Evolution and functions of human dance. Evolution and Human Behavior, 42(4), 351-360. https://doi.org/10.1016/j.evolhumbehav.2021.01.003

Fokin, M. (1962). “Against the current”. Memories of a choreographer. Moscow: Art.

Horrigan, K. (2020). Welcoming in dancers from all traditions. Journal of Dance Education, 20, $142-147$. https://doi.org/10.1080/15290824.2020.1789147

Karageorghis, C. I., Lyne, P. L., Bigliassi, M., \& Vuust, P. (2019). Effects of auditory rhythm on movement accuracy in dance performance. Human Movement Science, 67, article number 102511. https://doi.org/10.1016/j.humov.2019.102511

Kharakhonova, M. A. (2018). Features of communication in modern culture. Proceedings of Young Scientists and Specialists of the Samara University, 2, 79-83.

Miles, M. C., \& Fuxjager, M. J. (2018). Animal choreography of song and dance: a case study in the Montezuma oropendola: Psarocolius montezuma. Animal Behaviour, 140, 99-107. https://doi.org/10.1016/j.anbehav.2018.04.006

Mileshko, A. L. (2018). Civilizational specificity of the art of dance (East, West): Culture, art and philology: Modern views and scientific research. Culturology, art history and philology: modern views and research. Collection of articles based on the materials of the IX International Scientific and Practical Conference, 2018, 43-49.

Narozhnaya, V. D. (2017). Ethnocultural functions of iconic gestures in non-verbal communication dance Ethnocultural functions of sign gestures in non-verbal communicative dances. Journal Language: History and Modernity, 4, 94-104.

Orlandi, A., Cross, E. S., \& Orgs, G. (2020). Timing is everything: Dance aesthetics depend on the complexity of movement kinematics. Cognition, 205, article number 104446. https://doi.org/10.1016/j.cognition.2020.104446

Pakes, A. (2021). Choreography invisible: The disappearing work of dance. The Journal of Aesthetics and Art Criticism, 79(4), 524-528. https://doi.org/10.1093/jaac/kpab048

Pimenova, Z. V. (2015). Dance communication in the digital age. Scientific Bulletin of the Moscow State Technical University of Civil Aviation, 215, 122-126.

Romanova, N. (2020). Cross-cultural dialogue: Historical-cultural heritage and basic values (on the example of the city of Murom). Smart Innovation, Systems and Technologies, 138, 169-176.

https://doi.org/10.1007/978-3-030-15577-3_17

Sokol, I. A. (2007). Correlation of the concepts of communication and communication. Materials of the VII 
International Scientific-Practical Conference "Personality-Word-Society” (p. 61). Minsk: Parkusplus.

Somov, G. (2013). The interrelation of metaphors and metonymies in sign systems of visual art: An example analysis of works by V.I. Surikov. Semiotica, 2013(193), 31-66. https://doi.org/10.1515/sem-2013-0003

Tkachuk, M. A. (2012). Social dance in the system of intercultural communication. Oikumena. Regional Studies, 2(21), 29-35.

Volkova, A. A. (2016). Gypsy art as a way of communication with European culture connection with European culture. Collection of Materials of the International Scientific and Practical Conference "Art, Design and Modern Education", 2016, 358-368.

\section{Copyrights}

Copyright for this article is retained by the author(s), with first publication rights granted to the journal.

This is an open-access article distributed under the terms and conditions of the Creative Commons Attribution license which permits unrestricted use, distribution, and reproduction in any medium, provided the original work is properly cited. 